rev.relac.int.estrateg.segur.5(1):33-50,2010

\title{
PODER Y SISTEMA INTERNACIONAL: UN APORTE APÓCRIFO A LAS RELACIONES INTERNACIONALE`
}

Henry Cancelado Franco**

\begin{abstract}
RESUMEN
Las relaciones internacionales tradicionalmente se estudian de una manera positiva, tratando de objetivar la realidad a estudiar y creando esquemas de análisis cerrados. Los actores internacionales son observados como actores cerrados y racionales en el sistema internacional, sin embargo, es necesario entender las dinámicas internas de tales actores, y en qué momento hay un empoderamiento de uno u otro discurso, generando así una nueva estructura ideológica que, gracias a los procesos comunicacionales, cambian la estructura y la ideología en una época específica. Dos problemas quedan en el debate de las relaciones internacionales siempre: el papel de la soberanía y el papel de la economía. ¿Qué pasaría si ambas
\end{abstract}

\footnotetext{
*Este artículo es un producto de la investigación titulada «Dejando Westfalia», elaborada a lo largo del último año.

** Docente e Investigador de la Universidad Militar Nueva Granada, Facultad de Relaciones Internacionales, Estrategia y Seguridad. hcancelado@gmail.com
} 
van inextricablemente unidas en los procesos históricos de los últimos siglos, generando así un sistema internacional diferente al que usualmente se piensa?

Palabras Clave: Sistema internacional, poder, economía, saber, comunicación.

\begin{abstract}
International relations are traditionally studied in a positive way, trying to see objectively the reality to be studied and creating closed analysis schemes. International actors are perceived as closed and rational in the international system; nevertheless, it is necessary to understand the internal dynamics of such actors, and to perceive at what moment there is an empowering discourse that generates a new ideological structure that, through the communication processes, changes the structure and ideology in a specific period. Two issues always remain in the discussion of international relations: the role of sovereignty and the role of the economy. What would happen if both are inextricably linked in the historic processes of the last centuries, creating thus an international system different from the one usually considered?
\end{abstract}

Key Words: International system, power, economy, knowledge, communication.

\title{
INTRODUCCIÓN
}

Los cambios experimentados en el sistema internacional, en su estructura, desarrollo y cosmogonía, cambios similares a los acaecidos en el mundo en la transición entre la Edad Media y la Modernidad, hacen pensar en un mundo confuso y difuso, con elementos poco tangibles en algunas de sus aristas, tanto políticas como económicas y sociales, con una institucionalidad en revaluación y con tendencias que desafían el orden anterior. A pesar de las visiones que nos hablaban del fin de toda estructura de pensamiento y el éxito incuestionable de la forma capitalista con sus valores intrínsecos, propios de su desarrollo ético, podemos ver hoy que a la escena internacional han regresado con fuerza elementos que la Modernidad y el liberalismo jamás imaginaron que reaparecerían y que se pensaban sepultados cuando el experimento iluminador de los siglos XVII y XVIII daba por lente único para entender el mundo y a sus fenómenos a la razón y que generaba una nueva visión para el ser humano, distanciada de la superstición y de las formas precapitalistas de siglos anteriores.

Esta es una perspectiva que fácilmente se supera, no desde la exacerbación del discurso postmodernista, sino desde la rigurosa fenomenología del sistema internacional contemporáneo. Asistimos a formas recurrentes y anteriores, como la profundización de un sistema mundial capitalista ${ }^{1}$; algunos teóricos como Samir Amin hablan de la Mundialización, pero también asistimos

\footnotetext{
${ }^{1}$ Dentro de los estudios posguerra fría, parecen confundirse los conceptos occidente, globalización, mundialización, globalidad, imperio. Pero todos parecen confluir en unos valores comunes que hacen pensar en un solo "ente» que busca posicionarse en el mundo.
} 
a la eclosión de formas novedosas que hacen que el mundo parezca el resultado del amalgamiento de elementos premodernos, modernos y postmodernos y se presenten elementos particulares que crean una tensión dentro del mismo sistema. Hoy en día se puede hablar de modelos de desarrollo para acercarse a una formación capitalista moderna, pero a la vez un estudio de las relaciones internacionales puede adentrarse en el estudio de la díada inserción internacional-resistencia cultural que se presenta desde fenómenos como la globalización o la atropellada «glocalización».

Los análisis sobre la realidad internacional fluctúan entre la avasalladora perspectiva de la inserción económica de sectores productivos y el desarrollo del sistema financiero, y la esperanza escatológica de parte de los experimentos de tipo identitarios que salpican el discurso político y generan ideologías de resistencia. Esto nos da como resultado un sistema internacional escindido, separado de su realidad y que académicamente no se ha podido manejar de manera conjunta para obtener un mejor panorama de su realidad contemporánea.

En el mundo actual se habla de un enfrentamiento entre el sistema internacional y el Ilamado «eje del mal»; esta dicotomía en la realidad internacional hace que el globo se divida entre aquellos que creen asumir las formas correctas para poder actuar en la política internacional sin constituirse en amenaza para el discurso homogenizador que se presenta, y que obliga a asumir una escala axiológica que indefectiblemente sumará aliados con beneplácito, pues en ese eje pareciera encontrarse el polo único de poder del sistema, y que deja por fuera a aquellos actores que asumen la vida del sistema internacional desde una óptica diferente a la liberal política y mucho menos capitalista, es decir, negarían lo que Oliver Cox Ilamaría la confluencia del orden social, jurídico, religioso y científico hacia el comercio y el mercado, originando así un núcleo vital en la comunidad que es la economía. ${ }^{2}$

Lo que parece presentarse es una forma no económica de articulación de la vida, que en primer lugar fracciona al Estado nación y genera formas no nacionales de agremiación. En segundo lugar genera redes paralelas al sistema internacional capitalista, cuestionando la legitimidad de los polos de poder tradicionales y generando unas formas alternativas del mismo a nivel mundial, lo que conlleva a una división polar en el mundo y origina un desfase en el proyecto homogenizador de occidente. Este desfase consiste en que el discurso y la acción occidental parecen nutrirse de varios elementos que la legitimarían a nivel mundial, elementos de tipo ideológico, político, económico y militar. Pero no logra que estos elementos se materialicen de una manera uniforme, sino que este discurso se consume de manera correlativa y activa. ${ }^{3}$

Esto genera un poder paralelo que no necesariamente es homogéneo en sí, ni el efecto que busca es homogenizar como tal el sistema internacional, simplemente parece ser una amalgama de procesos que se yuxtaponen, pero que caen muchas veces en el discurso dominante y la mayoría de las

\footnotetext{
${ }^{2}$ COX Oliver, El capitalismo como sistema, Editorial fundamentos, Caracas, 1972. p. 37.

${ }^{3}$ Cfr. GARCÍA Canclini Néstor, Consumidores y ciudadanos, México, Grijalbo, 1995. p. 49.
} 
veces generan otras estructuras diferentes a las de dicho discurso. El poder paralelo se aísla de la dinámica internacional y las regiones entonces se distancian de los centros de poder, lo que origina que la participación de las regiones excluidas se tenga que hacer por otros medios y, a su vez, esta dinámica socava el poder establecido, generando transformaciones culturales e históricas en el sistema internacional contemporáneo.

De manera generalizada, los actores del sistema internacional han asumido una concepción economicista de las relaciones internacionales y se asume que el poder como tal debe ser estudiado y basado en los indicadores económicos. Esto hace que tales actores traten de seguir las fórmulas económicas, políticas y comerciales que les permitan presentarse como poderosos y merecedores de influencia en el sistema. La idea de inserción internacional ha llevado a profundizar las corrientes comerciales y, además, se han expandido y modernizado los sectores exportadores de muchas economías emergentes. ${ }^{4}$

Sin embargo, a pesar de los desarrollos en esta materia a nivel internacional, aparecen elementos adyacentes a la inserción económica que hacen que haya elementos culturales, identitarios y políticos que se proyectan lejos del esquema comercial y político convencional del sistema internacional. Estos elementos generan redes paralelas que le permiten a ciertos sectores poder aumentar su participación en un sistema mundial para no quedarse rezagados en el escenario internacional y aprovechar un clima de globalización que permite la atomización de las formas clásicas de poder al posicionar a nivel mundial actores paralelos que se empoderan de manera vertiginosa y difuminan los canales de poder mundial. De esta manera se originan los polos alternativos de poder en el sistema internacional.

El objetivo de este escrito es aportar al debate sobre el sistema internacional, desde la estructuración de poder en el mismo, partiendo del supuesto de que el poder internacional es capaz de transformarse y de generar, desde elementos que aparentemente son imperceptibles, nuevas formas en las relaciones internacionales. ¿Es este cambio un simple sofisma conceptual?, ese es el debate que se pretende plantear. Es necesario entender al sistema internacional como un organismo que tiene unas tendencias y que se enfrenta a contratendencias que buscan establecer formas ideológicas diversas para generar focos de poder que se comunican entre ellos y que originan una cantidad de redes significativas que permiten a los actores internacionales actuar de muchas maneras y determinar cambios o continuidades dentro del sistema.

\footnotetext{
${ }^{4}$ Cfr. FRENCH-DAVIS Ricardo, "Reformas económicas, globalización y gobernabilidad en América Latina» en MAGGI Claudio, MESSNER Dick (ed) Gobernanza global, una mirada desde América Latina, Nueva Sociedad, Caracas, 2202. p. 155.
} 


\section{ELEMENTOS DE UNA TEORÍA DEL PODER PARA LAS RELACIONES INTERNACIONALES}

Desde el Realismo posterior a la Segunda Guerra Mundial se proponía que lo determinante del sistema internacional era la lucha por el poder, con una clara tendencia a asumir la violencia organizada como una forma legítima de conseguir tal poder.

Dentro de la lógica realista, la racionalidad del Estado permite que este tenga una actuación coherente dentro del sistema, de acuerdo con sus intereses en el sistema internacional, el cual es anárquico. ${ }^{5}$ Adicionalmente, una característica del realismo supone a los estados como los únicos actores en el sistema; en esta estructura teórica, la fuerza es un elemento primordial y rentable en el desarrollo de las relaciones entre los actores del escenario mundial. Por causa de lo anterior, la agenda de los estados está jerarquizada, sobreponiendo el aspecto militar ante los demás. La política de la seguridad militar predomina sobre la política de los asuntos económicos y sociales.

La definición de sistema y política internacional, bajo la concepción realista, se enmarca en la protección continua de amenazas reales o potenciales. Esto da como resultado el uso continuo de la fuerza con el objetivo de alcanzar o prevalecer como líder militar en dicho sistema. Todo, a fin de obtener el poder necesario que le permita perseguir «libremente» el alcance de su interés nacional. Es por esto que la integración política y la cooperación no son elementos que aparezcan claramente en esta teoría.

El Neorrealismo o Realismo Estructural parte de unas concepciones menos pesimistas de las relaciones internacionales y le atribuyen una bondad ontológica y antropológica del ser humano a los actores internacionales, sin embargo, el elemento de fuerza se sigue manteniendo como un elemento de disuasión dentro de las relaciones internacionales. Los Estados van a la guerra por tener una deficiente organización social y es por esta precariedad social que el sistema internacional es denominado anárquico. Esta anarquía internacional no permite que los problemas de seguridad sean resueltos en el sistema debido al desorden. Kenneth Waltz supone que el sistema es anárquico «no solamente por la ausencia de gobierno, sino también por la presencia de caos y el desorden». ${ }^{6}$ La incertidumbre en el sistema nos lleva a una escalada en el incremento de los aparatos de defensa y a la exacerbación del discurso de securitización por parte de los Estados cuando se ven amenazados.

De acuerdo con esta visión neorrealista de los subsistemas, podemos afirmar que estos últimos son relevantes en la medida en que sean funcionales para las grandes potencias. En este momento se percibe una disminución de la anarquía debido a la formación de alianzas. Pero estas alianzas son ocasionales y solo sirven hasta que el objetivo de la potencia es alcanzado, luego el Estado o subsistema débil decrece en importancia dentro del sistema internacional.

\footnotetext{
${ }^{5} \mathrm{Cfr}$. WILHELMY von Wolff, Manfred, Política internacional: enfoques y realidades, Centro Interuniversitario de Desarrollo - Grupo Editor Latinoamericano, Buenos Aires, 1988. Cap. 2

${ }^{6} \mathrm{Cfr}$, WALTZ, Kenneth, Teoría de la Política Internacional. Buenos Aires, GEL, 1988.
} 
Así mismo, los Estados débiles procuran seguir los caminos recorridos de los Estados fuertes y poderosos en su accionar, a fin de alcanzar el liderazgo de estos. Pero es muy difícil lograr dicho objetivo, no sólo porque las grandes potencias ya se han ganado una reputación militar muy grande en el sistema, sino porque, como su nombre lo indica, estos «Estados débiles» son demasiado frágiles como para pretender enfrentar a una potencia mundial, y estas poco están interesadas en ellos a menos que sea para mantener su status e influencia mundial. Lo que le da a los subsistemas un estado de vulnerabilidad permanente. Para el Neorrealismo sí hay cabida en el sistema para otros actores, sin embargo, el Estado sigue siendo el principal y más poderosos actor en el escenario global. Y estos otros actores existirán, así como los Estados débiles o subsistemas, para permitir el alcance de los intereses de las superpotencias.

Si observamos detenidamente estas teorías, ninguna sirve para analizar correctamente a otros actores del sistema internacional, si bien se inscriben en las relaciones de poder que maneja el sistema internacional. ${ }^{7}$ Para lograr solucionar esta aparente falla es necesario adentrarse entonces en un estudio teórico del concepto poder. Lograr abrir este concepto y llevarlo más allá de la simple instrumentalización constructivista para poder entender que no es un concepto de suma cero y que en esa aparente anarquía hay lógica y múltiples actores.

\section{GENEALOGÍA DEL PODER}

Hay que estudiar, actualmente, la forma en la que se estructura el poder a nivel global. El sistema internacional es una macro expresión de lo que ocurre a nivel de una microfísica social, los Estados son los sujetos de esta macro expresión en la que se entremezclan diversos poderes y que cuestionan, amenazan o refuerzan concepciones teóricas clásicas que quedan obsoletas para el mundo contemporáneo. Establecer nuevos elementos de seguridad y nuevas razones desde las cuales se estudia el sistema internacional es el deber de los estudiosos de las relaciones internacionales. Crear nuevos elementos y rehacer genealogías para poder entender un sistema internacional lejano de Westfalia, y con nuevos retos y elementos, es una labor ardua de la que depende el futuro de las relaciones internacionales como ciencia; rehacer una episteme que lleve a la ciencia por nuevos campos es la labor a desarrollar nuevos conceptos y nuevos enfoques que hagan de la teoría de las relaciones internacionales algo rico epistemológicamente y amplio en cuanto campo de estudio.

El Estado sigue siendo, sin lugar a dudas, el actor por excelencia del sistema internacional, pero ignorar el relativismo de su poder contemporáneo es ignorar nuevas variables de poder que entran a determinar el juego de los actores en el sistema internacional. Por eso es necesario entrar a estudiar el concepto «poder» en primer lugar, redefinirlo y cargarlo de nuevos componentes, de lo contrario, se estaría analizando una realidad diferente con herramientas conceptuales obsoletas para el contexto actual.

\footnotetext{
${ }^{7}$ Cfr. AYOOB Mohammed, «The Third World in the System of States: Acute Schizophrenia or Growing Pains?» En International Studies Quarterly, Vol. 33, No. 1, March 1989. pp. 67-79.
} 
Usar la genealogía para realizar un análisis requiere ocuparse de aquellas cosas aparentemente ocultas, aquellas verdades escondidas tras máscaras que traen en sí mismas el origen para, de esta manera, captar la individualidad de los sucesos, aquello que lo hace singular o diferente, encontrando la esencia exacta de cada cosa.

Para encontrar el origen del instrumento de nuestro análisis es preciso hallar su fuente de procedencia, lo cual nos permite encontrar la diversidad en lo que posteriormente se convertirá en unidad, mostrando el cómo y el dónde surgen los fenómenos que permiten la aparición del punto de emergencia o punto de surgimiento. Este punto de emergencia se produce siempre en un determinado estado de fuerzas que luchan por imponerse, por anularse o por dominar. La emergencia se produce en el intervalo o espacio que media entre dos cuerpos, dando como resultado una escena en la que es posible apreciar la distribución de un grupo de sujetos frente a otros en un espacio en el que intercambian amenazas, mostrando el eterno juego entre los dominadores y los dominados, y este juego es el que permite fijar los niveles de diferenciación entre los sujetos y la posterior delimitación de su papel en el sistema. De esta manera, la humanidad ha venido enfrentando diversos procesos de dominación, instaurando reglas que sustituyen la guerra y garantizan el ejercicio de los mismos. Por ejemplo, los procesos ideológicos-institucionales posteriores a la Segunda Guerra Mundial, como Bretton Woods, GATT y, por qué no, la misma ONU, organizando una idea de cooperación y entendimiento mundial.

Por otro lado, se asume que el poder es información, es conocimiento, es saber y «debe su fuerza a la dureza que lo opone a lo que lo rodea; y es mediante la aparición de este saber, de estos saberes locales de la gente, de estos saberes descalificados como se ha operado la crítica». ${ }^{8} \mathrm{De}$ todas formas hay una instrumentalización del saber en torno a una dialéctica de poder, de enfrentamientos en lo cotidiano que estructura unas relaciones sociales. Tales enfrentamientos crean el saber que se convierte en poder; sin embargo, queda la pregunta en el aire de si el poder que se origina se circunscribe exclusivamente al savoir faire de las relaciones de producción.

Por lo tanto, denominamos genealogía a la unión de los contenidos históricos y las memorias locales «que permite la constitución de un saber histórico de la lucha y la utilización de ese saber en las tácticas actuales» ${ }^{9}$. La genealogía del poder tiene como finalidad emprender una lucha contra los efectos del saber centralizador, que es empleado en las instituciones de nuestra sociedad y pretende filtrar los saberes no legitimados en nombre del conocimiento «verdadero» y unas posturas reales y ortodoxas.

En este contexto podemos apreciar cómo la genealogía se convierte en un mecanismo que nos acerca a realizar un análisis del poder mediante cuestionamientos tales como ¿Qué es el poder?, se pregunta Michel Foucault, y se remonta al S. XVIII, en donde el poder era visto como un derecho

\footnotetext{
${ }^{8}$ FOUCAULT, Michel. Microfísica del poder. Madrid. Las Ediciones de la Piqueta, 1980. p. 129.

${ }^{9}$ Ibíd. p. 130.
} 
que puede transferirse total o parcialmente mediante un acto jurídico para, de esta manera, contribuir a la constitución de un poder político y, por ende, a una soberanía.

Esta concepción jurídica y liberal del poder encuentra un punto común con la teoría marxista, mostrando la funcionalidad económica del poder, en donde este busca mantener las relaciones de producción y dominación de clase que permite su libre desarrollo y la apropiación de la fuerza productiva que lo hace posible.

Pero en un contexto más moderno, ¿Qué disponemos actualmente para el análisis del poder? Teniendo en cuenta esto, podemos ver cómo el poder sólo existe en la medida en que se lleva al ejercicio a través de una relación de fuerzas y, sin lugar a dudas, esto nos acerca a otros cuestionamientos: ¿Qué implica este ejercicio?, ¿Cómo llevarlo a cabo?, ¿Cuál es su estructura de funcionamiento? De esta manera el poder es trabajado como un mecanismo de represión.

Sin embargo, «¿No debería ser analizado el poder en términos de lucha, de enfrentamientos, de guerra?» ${ }^{10}$

Para responder este interrogante es necesario ver que en una sociedad las relaciones de poder funcionan mediante una vinculación de fuerzas establecida en un momento determinando de la guerra. En el momento en que la guerra culmina se pretende hacer ver al poder político como un mecanismo que suspende los efectos de la guerra; sin embargo, el papel del poder político iría más allá y emprendería un papel oculto que mantendría latente los efectos del enfrentamiento por medio de las instituciones, las desigualdades económicas, entre otros, Ilegando a la conclusión de que el término de la política suspendería el ejercicio del poder como una guerra continua.

De esta manera, tendríamos dos puntos de análisis del poder: por un lado encontraríamos el ejercicio de la opresión a través del contrato social, que es de tipo jurídico, y por otro encontraríamos un esquema de represión a través de la guerra que muestra al máximo el ejercicio de la lucha y la sumisión. En el sistema internacional es la lucha por la inserción o por mantener una resistencia y una identidad no homogenizadas en el discurso totalizante del sistema contemporáneo.

En este orden de ideas es importante el establecimiento de unas normas que permitan resolver las contradicciones que se dan en el seno del mundo, pues se trata de un acto de justicia y manejo de la política internacional que alcance su plenitud y para ello hay que reconocer que lo hará en la medida en que pueda controlar a la mayor cantidad de Estados y actores al interior de sí mismo.

Como es bien sabido, el Estado no es la sede privilegiada y exclusiva del poder, debido a que este se expande a través de diversos mecanismos que tienen como finalidad llegar al individuo.

\footnotetext{
${ }^{10}$ Ibíd. p. 135.
} 
El poder no se construye a partir de la unión de voluntades individuales o colectivas; el poder se construye y funciona a través de efectos del poder que posiblemente no encontraremos en el Estado, sino más bien en las familias, las instituciones educativas, las prisiones, los centros médicos, en el sistema productivo, etc. En el sistema internacional encontramos toda serie de dispositivos de control, exclusión, normalización y represión que tienen como finalidad moldear o transformar al Estado convirtiéndolo en sujeto, es decir, en un ente adaptado al sistema e implícitamente convertirlo en objeto de dominación, con la finalidad de conseguir un sistema disciplinado y organizado.

Es así que podemos concebir al poder como una red de micropoderes dispersos por todo el sistema internacional, sin un punto central y coordinado por instituciones. El ejercicio del poder va mucho más lejos que el Estado; pasa por canales más finos que permiten que el poder circule y que cada individuo en el fondo detente cierto nivel de poder.

Para realizar un análisis de las relaciones de poder debemos concebirlo como algo que circula o funciona en cadena. Nunca está localizado en un lugar específico o en manos de algún grupo. El poder funciona de manera transversal y en sus redes no sólo se encuentran los Estados, sino que, a su vez, estos se encuentran en la posibilidad de sufrir o ejecutar este poder. Al interior de la sociedad las relaciones de poder atraviesan, caracterizan y constituyen el cuerpo social. Estas redes de poder no pueden dividirse debido a que interconectan el sistema.

En el interior de la sociedad es posible encontrar diversos mecanismos que permiten el ejercicio del poder por medio de tácticas represivas, con la finalidad de preservar el predominio de la clase dominante. Entre estos estilos de represión podemos encontrar la que se lleva a cabo a través de los institutos, como es el caso de una institución educativa que reprime a nivel grupal a través de instrumentos como la enseñanza o la información orientada.

El saber transmitido mediante la enseñanza usualmente toma una apariencia positiva y de rápida aceptación, pero en realidad funciona como un mecanismo de exclusión-represión, en el sentido que regula los niveles de información, los cuales deben ser transmitidos al interior de la sociedad, excluyendo a aquellos que no tienen derecho a saber o que solo les puede ser revelado un determinado tipo de saber, formando "lo que podría llamarse los «circuitos reservados del saber», aquellos que se forman en el interior de un aparato de administración o de gobierno, de un aparato de producción, y a los cuales no se tiene acceso desde afuera» ${ }^{11}$.

El saber académico exige un determinado sistema de enseñanza e implica una determinada conformidad política en la que la historia de las luchas por el poder y, en consecuencia, las condiciones reales de su ejercicio y de su sostenimiento siguen estando casi totalmente ocultas.

\footnotetext{
${ }^{11}$ Ibíd. p. 32.
} 
Para llevar a cabo la represión, a través de la educación, el humanismo es uno de sus instrumentos, debido a su influencia en el aprendizaje y su aporte al mantenimiento de la organización social. El humanismo, teniendo en cuenta que es un discurso Occidental, es entendido como el conjunto de discursos que buscan infundir en la mentalidad del individuo occidental la idea de soberanía sin detentar el poder, pero subordinada a un mecanismo de represión, creando así soberanías sometidas, tal como sucedería en el campo espiritual: el alma, que es soberana sobre el cuerpo, es sometida a Dios; o en el campo material, donde el individuo es soberano de sus derechos, pero se encuentra sometido a las reglas de la sociedad. Acabar con la represión del humanismo implica realizar una destrucción del modelo cultural, que mostraba al individuo como pseudo-soberano, es decir, todos esos mecanismos de exclusión social, exclusión intelectual o simplemente ruptura de todas las prohibiciones que de cierta manera ejercían represión.

Sin embargo, la enseñanza no es el único medio del que se apoyan el humanismo y la represión social para llevar a cabo su finalidad, es necesario que visualicemos otros mecanismos de transmisión del saber, como por ejemplo, los periódicos y la televisión, que influyen en la conciencia del individuo encaminando su percepción de la realidad y filtrando la información. Igualmente, se utilizan mecanismos de represión de tipo moral, como es el caso de la lucha contra las drogas o los centros de reclusión para enfermos mentales que, aparte de ejercer labores de vigilancia sobre estos «inadaptados» sociales, son los encargados de mostrar el prototipo del hombre normal, racional y adaptado a la comunidad.

Teniendo en cuenta este esquema que trazan unos sobre otros para mantener su predominio en el sistema, que varían desde la educación vigilada hasta la represión psicológica en la elección de sus estudios y sus categorizaciones de tipo axiológico, se llega a la pregunta sobre el poder del discurso liberal occidental y economicista que ahora se vive en el sistema internacional. ¿Homogeniza al mismo sistema, lo fractura de tal manera que lo lleva al borde de su propia anarquía, o simplemente difumina en nuevas redes de participación internacional?

\section{MECANISMOS DE PODER, VIGILANCIA Y CONTROL}

Para comprender la forma en la que funciona el poder, es necesario pensar en un sistema de redes que se expande a través del cuerpo social y llega a los individuos, insertándose en su interior, manipulando e imponiendo su papel en el sistema. Esto se debe a una serie de cambios que se han venido presentando desde el siglo XVIII, en donde podemos apreciar el surgimiento de un «poder sináptico», es decir, un poder que interconexiona todos los elementos del cuerpo social, lo que hace que el poder del soberano sea relegado o se le reste importancia.

Para que este tipo de poder mantenga su predominio en el sistema es necesario que se instauren toda una serie de mecanismos que permitan mantener vigilada la acción del individuo, como es el caso de las prisiones o las instituciones educativas, en donde se busca moldear o transformar al individuo en una persona adaptada a la comunidad. Sin embargo, lejos de transformar o moldear al individuo en una "persona de bien» o «apta» para la sociedad, lo que se hace es convertir al 
individuo en un sujeto del sistema, por lo cual estos mecanismos de vigilancia y control que se instauran en las instituciones se encargan de asignar a cada individuo su papel en el sistema de manera estratégica con la finalidad de mantener el equilibrio. Es así que nos es posible entender el porqué muchos criminales continúan delinquiendo aún después de permanecer largo tiempo en las prisiones, y las prisiones, «lejos de transformarlos en gente honrada, no sirven más que para fabricar nuevos criminales o para hundirlos todavía más en la criminalidad». ${ }^{12}$ Es por esta razón que en el interior de cada sociedad encontramos tantos delincuentes, como jueces y educadores que son útiles al sistema, pues encaminan el desarrollo del dominio económico y político de la sociedad.

Por otra parte, podemos encontrar otro tipo de mecanismo del poder: el saber. Es necesario entender que el poder y el saber se articulan, debido a que ejercer el poder crea objetos del saber y el saber conlleva efectos del poder. El saber es un mecanismo a través del cual el Estado hace que circule la información que considera pertinente y restringe determinados saberes que de cierta manera desequilibran su estructura. El saber circula en el sistema social a través de las instituciones educativas y medios de comunicación masiva que influyen en la conciencia del individuo y encaminan su percepción de la realidad. El saber opera sobre el individuo, gracias a la imposición de diversas disciplinas escolares y militares que garantizan la obediencia a través de un conjunto de comunicaciones reguladas y la diferenciación del papel de cada individuo.

La finalidad de los mecanismos de vigilancia y control es proteger la estructura del Estado a través de la «eliminación» de los enfermos, el control de los contagiosos y la exclusión de los delincuentes, pero esta acción solo se legitima en la medida en que estos mecanismos de poder son llevados a cabo por medio de las normas del derecho.

Sin lugar a dudas, el derecho es el mecanismo más eficaz del poder, por medio del cual el Estado ejerce su labor de vigilancia y control, pues establece un tipo de «verdad» impuesta por el sistema, representada en normas, leyes y saber transmitido en forma de discurso que, a su vez, transmite efectos específicos del poder que tiene por objetivo transformar al Estado en sujeto y asignarle su papel dentro del sistema, en el que posteriormente es diferenciado y obligado a competir en búsqueda de su supervivencia. El derecho es el sustento político y económico del sistema internacional contemporáneo, es la máxima expresión de la modernidad occidental y hacia él se dirigen todos los esfuerzos por «organizar el globo».

Sin embargo, más allá de su papel de legitimación del poder, podemos ver que el derecho se enfrenta a un problema: la soberanía. Al decir que el problema central del derecho es la soberanía hacemos referencia a la función del derecho en disolver la concepción de dominación y en su lugar hace aparecer dos cosas: «Por una parte, los derechos legítimos de la soberanía y, por otra, la obligación legal de la obediencia». ${ }^{13}$ Este sistema de derecho se encuentra centrado en la figura del Estado que enmascara la dominación y sus consecuencias.

\footnotetext{
12 Ibíd. p. 90.

${ }^{13}$ Ibíd. p. 141.
} 
El papel del derecho va más allá de instaurar legitimidad; también se encarga de poner en marcha procedimientos de sometimiento. El derecho se convierte en un instrumento de la dominación. Estas relaciones de dominación no son de tipo global, es decir, de un gran líder sobre los demás individuos; esta dominación tiene múltiples formas de ejercerse en la sociedad, a través diversos sometimientos y obligaciones impuestos en el cuerpo social.

Para comprender esta dinámica, es necesario encontrarlo en el momento en el que circula a través del sistema internacional, en ocasiones incumpliendo con las normas de derecho que lo establecen y delimitan extendiéndose más allá de ellas, dando poder a las instituciones y, a su vez, adaptando técnicas e instrumentos materiales que incluso pueden Ilegar a ser violentos.

Para realizar un análisis crítico del poder es necesario dirigir la investigación no sobre el edificio jurídico de la soberanía y las ideologías que manejan; este análisis se debe orientar hacia la dominación, hacia sus instrumentos o las formas de sometimiento. Para entender el poder se requiere que lo estudiemos no centrándonos en el Estado y sus instituciones. El poder se debe estudiar desde las técnicas y tácticas de dominación, que son necesarias para que el Estado tenga un óptimo desempeño.

\section{EL PODER Y LOS NUEVOS ACTORES DEL SISTEMA INTERNACIONAL}

Es indudable que para entender las relaciones de poder se piense en un campo de negación del mismo, representado en las resistencias que emergen en el interior de las diferentes culturas. Estas resistencias se han desarrollado como una oposición al poder dominante en los diferentes círculos sociales que, a pesar de tener un carácter individualizante, debido a la especificidad de sus luchas antiautoritarias tienen elementos comunes que les permiten facilitar su análisis.

Estas luchas antiautoritarias son de tipo transversal, debido a que, aunque se pueden desarrollar más fácilmente en determinados países, no son propias de una sola región. Por ejemplo, las luchas del islamismo fundamentalista que se dan desde Filipinas, pasando por Timor Oriental y terminando en los Balcanes. Estas luchas tienen como finalidad la oposición al ejercicio del poder que impone obediencia y subordinación y que en muchas ocasiones está asociado con el conocimiento, la competencia y la calificación, por lo que se convierten en luchas contra el régimen del saber, en la medida en que hay una oposición en contra de los privilegios que este otorga. Generalmente, estas luchas adquieren un carácter «inmediato», buscando la oposición al poder que tenga influencia directa sobre los individuos en un periodo específico de tiempo, sin inquirir en una solución radical al problema de la dominación. Este tipo de resistencias son luchas que cuestionan el estatuto del individuo, porque mientras «afirman el derecho a ser diferentes y subrayan todo aquello que hace verdaderamente individual al individuo, atacan todo lo que separa al individuo» ${ }^{14}$ convirtiéndose

\footnotetext{
${ }^{14}$ FOUCAULT, Michel. «El Sujeto y el Poder». En: Revista Otras Quijotadas No. 2, Septiembre 1985. Medellín, p. 89.
} 
en luchas contra el Gobierno de la individualización, o contra el discurso homogenizador occidental que permite que en determinados momentos se marquen diferenciaciones que impliquen el ejercicio de la dominación, como es el caso de las estratificaciones sociales, las diferencias culturales o, incluso, económicas.

Este tipo de luchas que surgen con la resistencia tienen la finalidad de atacar toda aquella forma de poder que de una u otra forma delimita el papel del individuo en el sistema, imponiéndole todo tipo de dictámenes que tiene que reconocer y que los otros tienen que reconocer en él, convirtiéndolo así en sujeto. Por lo tanto, en el momento en que el individuo se transforma en sujeto, adquiere participación en el sistema, debido a que acepta el papel que le es impuesto y los niveles de diferenciación que lo separan de los otros individuos. De esta manera podemos identificar tres tipos de luchas a las que los individuos se enfrentan constantemente, como lo son las luchas contra las formas de dominación, contra las formas de explotación y las luchas contra las formas de sujeción (sumisión de la subjetividad), que ligan al individuo con su propio ser asignándole un papel en el sistema, que al mismo tiempo lo diferencia y lo somete a otros.

Actualmente las luchas contra las formas de sujeción adquieren relevancia, a pesar de que las luchas contra la formas de dominación y explotación no han desaparecido, y esto se debe a que los mecanismos de sujeción se relacionan directamente con ellas. Cabe advertir que esta clase de luchas tienden a permanecer vigentes, gracias a la existencia del Estado.

En este orden de ideas, el Estado es visto como una estructura de poder político que tiende a ignorar a los individuos para ocuparse solamente del interés general de la comunidad; sin embargo, el Estado combina en sus estructuras políticas técnicas de individualización y de totalización, gracias a que asume una técnica de poder denominada poder pastoral. El poder pastoral es un tipo de poder originado en las instituciones cristianas que, mediante el conocimiento perfecto y la habilidad para dirigir la conciencia de la multitud, se ocupa de la comunidad en general sin descuidar al individuo en particular, para de esta manera garantizar su salvación en el otro mundo. Es así como este poder implicaba el sacrificio del pastor por el bien de la comunidad.

En un contexto actual podemos observar que el Estado ha adaptado el poder pastoral en su estructura de funcionamiento, por medio de mecanismos e instituciones a su servicio que se extienden en todo el cuerpo social con la finalidad de satisfacer las necesidades básicas de la comunidad, garantizando su supervivencia. Este poder totalizador del Estado, que tiene como objetivo regular las relaciones de poder dentro de la sociedad, actúa mediante una táctica individualizante en la que delega parte de su poder a multiplicidad de instituciones como la familia, la medicina o la educación, con las que llega más eficazmente a todos los individuos. Cabe observar que esta forma de poder en determinados momentos se puede llegar a convertir en un mecanismo que garantiza el control y la vigilancia del Estado sobre los individuos, de manera directa o indirecta, para poder limitar las resistencias que puedan surgir en la comunidad y con ello mantener las formas de dominación y explotación que dan origen a las luchas contra las formas de sujeción. 


\section{¿CÓMO SE EJERCE EL PODER?}

El poder comprende un conjunto de acciones que ponen en juego las relaciones entre los individuos. El ejercicio del poder supone la interrelación de las relaciones de poder, comunicación y las capacidades objetivas que, aunque no deben confundirse, se complementan entre sí para llegar a un fin específico.

Para el poder totalizante del sistema resulta casi imposible lograr una coordinación uniforme de estos tres tipos de relaciones, debido a que «en una sociedad dada no existe un tipo general de equilibrio entre las actividades finalizadas, sistemas de comunicación y las relaciones de poder», ${ }^{15}$ por consiguiente, el Estado busca medios específicos y circunstancias determinadas en las que estas interrelaciones se establecen de acuerdo con un modelo preestablecido, que logra su ejecución a través de instituciones, constituyéndose en sistemas organizados. Ejemplo de lo anterior es una institución educativa, en donde la relación capacidad-comunicación-poder se logra constituir de manera complementaria, de forma tal que la actividad del aprendizaje se desarrolla partiendo de un conjunto de comunicaciones reguladas (que se manifiestan a través del sistema de preguntas y respuestas, obediencia y diferenciación del papel de cada individuo) y procesos propios del poder como la vigilancia, el castigo o la recompensa.

Las instituciones en donde se logra llevar a cabo el ejercicio de la relación capacidad-comunicaciónpoder, constituyen medios para lograr establecer disciplinas y con ello garantizar la obediencia e implantar sistemas de vigilancia y control del Estado sobre el individuo, o del sistema sobre los actores internacionales.

\section{CONSTITUCIÓN DE LA NATURALEZA ESPECÍFICA DEL PODER}

El poder no es tangible, solo existe en la medida en que es puesto en acción; sin embargo, no podemos limitar la definición del poder a una acción que actúa de manera directa sobre los individuos, debemos ir más allá y verlo como una acción que actúa sobre las acciones de los individuos, lo que significa que es una manera de actuar sobre los sujetos en contraposición a su capacidad de acción, teniendo la posibilidad de guiar su conducta y contrarrestar la posible consecuencia de las resistencias.

Por lo tanto, para que sea viable el ejercicio del poder se requiere el «gobierno» ${ }^{16}$ de los hombres por otros hombres, buscando la posibilidad de construir un campo en el que sea posible vigilar la acción de sus semejantes. Al definir el poder como una acción paralela a las acciones de los

\footnotetext{
${ }^{15}$ Ibíd. p.96.
}

${ }^{16} \mathrm{El}$ término gobierno, en este sentido, sería empleado como la capacidad de dirigir la conducta del individuo o de los grupos de individuos. 
individuos y con ello materializarse en el gobierno de los hombres sobre otros hombres, nos es necesario recurrir a otro término: libertad.

La libertad y el poder tienen una relación directamente proporcional, debido a que el poder sólo se ejerce sobre sujetos libres que tengan capacidad de acción, convirtiendo a la libertad en la condición previa al poder y el garante de su funcionamiento, dado que, sin la posibilidad de la desobediencia, la necesidad del poder sería nula y pasaría a convertirse en una relación de represión.

En el caso de muchos actores, el poder y la estructuración que se ha hecho de sí mismo en el sistema internacional, desde los discursos dominantes y desde la institucionalidad internacional, ha intentado ocultar las especificidades culturales, políticas y económicas del sistema internacional, forzándolos a entrar en ese juego dialéctico. Sin embargo, la resistencia no se hace esperar y la diversidad latinoamericana es más fuerte que la homogenización en torno a una idea del sistema internacional; la eclosión de nuevas redes y variables de poder hacen que los actores se puedan comunicar por algunos canales paralelos a los oficiales con el resto del sistema; el sistema vive en caos y los actores se aprovechan de esta situación, el poder explota y se reparte constantemente entre diversos actores y canales de participación a nivel internacional.

\section{LA ECONOMÍA Y EL PODER}

Existe un problema cuando se analiza el poder, y es que si este parte desde un entorno jurídico, ¿Cómo pasa a un entorno político? El Estado del siglo XVIII, y antes, estructuraba todas sus relaciones de poder en torno al derecho, a los derechos de sucesión y a los derechos de posesión, ${ }^{17}$ de tal manera que el paso de un Estado jurídico a un Estado político, como el que se pretendió en la creación de la nación como tal a finales del mismo siglo, se da gracias a un paso histórico esencial que, por supuesto, las teorías de las relaciones internacionales no tratan ampliamente. La organización del Estado en poder internacional se da en el momento de la expansión de los mercados globales, en la cual los imperios transatlánticos cambian su naturaleza netamente jurídica en una naturaleza política mediada por los intereses económicos. La expansión europea en África y Asia marca el inicio de una situación internacional, diferente a los imperios clásicos como el español, el cual, en efecto, pierde el poder a lo largo del siglo XIX, dando ascenso a nuevos Estados de otro tipo de poder en el sistema internacional: Alemania, Estados Unidos y Japón.

Este nuevo poder también estaba enmarcado en el cambio de relaciones de producción de una sociedad conservadora, basada exclusivamente en la tenencia de tierras, en la cual el concepto de soberanía era básico y claro, a una forma en la cual la expansión territorial se hacía para aumentar la producción de la metrópoli y hacerla competitiva a nivel internacional, abandonando la vieja

\footnotetext{
${ }^{17}$ Cfr. FOUCAULT Michel Seguridad territorio y población, clase del 1 de febrero de 1978. Buenos Aires, Fondo de Cultura Económica, 2006.
} 
forma de extracción para enriquecimiento exclusivo del centro colonial. De esta manera podemos hablar de un gobierno económico.

La palabra «economía» designaba una forma de gobierno en el siglo XVI, y designará en el siglo XVIII un nivel de realidad, un campo de intervención para el gobierno, a través de una serie de procesos complejos y, creo, absolutamente capitales para nuestra historia. Eso es, entonces, gobernar y ser gobernado. ${ }^{18}$

El poder necesita un puente transmisor de los derechos patrimoniales, que en definitiva son la primera forma de gobierno que existe, hacia las formas políticas de poder. La economía es ese puente transmisor y la influencia ${ }^{19}$, ya no sólo jurídica, sino también política y militar sobre la esfera económica. El problema en este punto es que, como consecuencia de este análisis, las relaciones internacionales como ciencia quedan supeditadas, ya ni siquiera a la ciencia política, sino a la economía política. La soberanía queda supeditada entonces a los intereses de varios soberanos con intereses económicos y no a la genialidad estratégica y política del estadista. Es decir, Maquiavelo fue superado, pero se quedó en el aire el problema de la soberanía; la economía tampoco fue capaz de establecer el principio soberano, por eso, ante las tensiones actuales de una economía globalizada, la soberanía sigue estando en el centro del debate. Por consiguiente, la economía política también queda corta. ¿Por qué es importante el problema de la soberanía? Porque de allí emana la concepción ideológica de poder que, al final de cuentas, es la que moviliza masas para uno u otro objetivo. Es decir, se cae en la tautología economía-política, sin encontrar una fácil solución.

\section{CONCLUSIONES}

Las teorías de las relaciones internacionales no se adentran en el problema del poder, lo mencionan tangencialmente sin hacer un proceso etimológico y epistemológico al mismo. Es necesario sacar el concepto de poder de la instrumentalización pragmática a la que ha sido sometido y encontrar su naturaleza y fuentes, para poder interpretar las diferentes lógicas y procesos que utiliza.

Es importante establecer nuevos elementos de análisis y nuevos conceptos y acercamientos teóricos desde los cuales se estudia el sistema internacional. De tal manera se puede entender un sistema internacional lejano de aquel orden del Tratado de Westfalia y con nuevos retos y elementos. Es un imperativo rehacer una episteme que lleve a la ciencia por nuevos senderos en el mismo campo del saber.

\footnotetext{
${ }^{18}$ Ibid, pág 121

${ }^{19}$ No se puede hablar de intervención en el sentido keynesiano del término, pues para esa época esa concepción iría en contra de los preceptos liberales decimonónicos; sin embargo, sí hay un manejo de la economía, tal y como lo vemos en Gran Bretaña y sus colonias, en la Conferencia de Berlín de 1885 y en la expansión estadounidense.
}

PODER Y SISTEMA INTERNACIONAL: UN APORTE APÓCRIFO A LAS RELACIONES INTERNACIONALES* 
En el sistema internacional se encuentran una serie de elementos de materialización del poder para poder «entificar» a los actores internacionales, de tal manera que se le convierte en un sujeto de dominio o de resistencia en el sistema.

El derecho era la herramienta, par excellence de dominio y gobierno; sin embargo, con la aparición de los conceptos políticos y con el cambio en el sistema económico y social en el siglo XVIII, el derecho se vio relativizado como fuente de poder, pasando a ser un instrumento del mismo, pero perdiendo sus elementos originadores de poder, y dentro del ejercicio del poder se requiere del gobierno de los hombres por otros hombres, buscando la posibilidad de construir un campo en el que sea posible vigilar y encauzar la acción de sus semejantes.

El poder necesita una conexión entre el derecho y la política, entre los jueces y el soberano; la economía es ese canal no sólo jurídico, sino también político y militar sobre la sociedad.

El problema de la soberanía sigue sin resolverse, es la fuente del poder en sí, sin embargo, cambia de acuerdo con las eras, pero es fundamental para entender en dónde se ejerce el poder o hacia dónde se encaminan las resistencias.

\section{BIBLIOGRAFÍA}

- $\quad$ AYOOB Mohammed, «The Third World in the System of States: Acute Schizophrenia or Growing Pains?» En International Studies Quarterly, Vol. 33, No. 1, March 1989. pp. 67-79.

- $\quad$ BALDWIN, David A. Neoliberalism neorealism, and World politics. Neoliberalism neorealism: term of the contemporary debate. University Press, New York, 1993.

- $\quad$ BRAUDEL Fernand, Las civilizaciones actuales, Madrid, Tecnos, 1971.

- $\quad$ CHOMSKY Noam, La estructura emergente del orden mundial, Bogotá. Universidad Nacional de Colombia, 2002.

- $\quad$ COX Oliver, El capitalismo como sistema, Editorial fundamentos, Caracas, 1972. p. 37.

- FAZIO Hugo, «La globalización, una reflexión desde la historia», en Globalización: discursos, imaginarios y realidades, Bogotá, IEPRI. págs. 13 - 45

- $\quad$ FOUCAUlT, Michel. Microfísica del poder. Madrid. Las Ediciones de la Piqueta, 1980. septiembre 1985.

„El Sujeto y el Poder». En: Revista Otras Quijotadas No. 2, Medellín,

Seguridad, territorio y población, clase del 1 de febrero de 1978. Buenos Aires, Fondo de Cultura Económica, 2006. 
- $\quad$ FRENCH-DAVIS Ricardo, «Reformas económicas, globalización y gobernabilidad en América Latina» en MAGGI Claudio, MESSNER Dick (ed) Gobernanza global, una mirada desde América Latina, Nueva Sociedad, Caracas, 2202.

- $\quad$ GARCÍA Canclini Néstor, Consumidores y ciudadanos, México, Grijalbo, 1995.

- $\quad$ HELD David. Transformaciones globales, México. Oxford. 2002.

- HUNTINGTON Samuel, ¿Quiénes somos?, los desafíos a la identidad nacional estadounidense, Bogotá, Paidós, 2004. págs. 339 - 416.

, El Choque de las Civilizaciones, Barcelona, Paidós, 1997.

- $\quad$ KENNEDY Paul, Auge y caída de las grandes potencias, Plaza y Janes, Barcelona, 1992.

- PEARSON Frederic, ROCHESTER, J Martin, Relaciones Internacionales, Mc Graw Hill, Bogotá, 2001.

- $\quad$ POMERANZ Kenneth. «The Great Divergente: China, Europe, and the making of the modern world economy» New Jersey, Princeton University, 2000.

- $\quad$ WALTZ, Kenneth, Teoría de la Política Internacional. Buenos Aires, GEL, 1988.

- $\quad$ WALLERSTEIN, Immanuel. Un mundo incierto. Buenos Aires. Libros del Zorzal. 2005.

- WILHELMY von Wolff, Manfred, Política internacional: enfoques y realidades, Centro Interuniversitario de Desarrollo - Grupo Editor Latinoamericano, Buenos Aires, 1988. 